\title{
Hyaluronic acid as a treatment for ankle osteoarthritis
}

\author{
Shu-Fen Sun · Yi-Jiun Chou • Chien-Wei Hsu • \\ Wen-Ling Chen
}

Published online: 13 March 2009

(c) The Author(s) 2009. This article is published with open access at Springerlink.com

\begin{abstract}
Viscosupplementation refers to the concept of synovial fluid replacement with intra-articular injections of hyaluronic acid (HA) for the relief of pain associated with osteoarthritis (OA). Intra-articular viscosupplementation was approved by the Food and Drug Administration (FDA) in 1997. It is currently indicated only for the treatment of pain associated with knee OA. However, OA can occur in several of the weight-bearing joints of the foot and ankle. Ankle OA produces chronic disability that directly impacts the quality of life. There is only limited published literature relating to the use of HA in the ankle. This paper will review the authors' experience, indications, clinical outcomes, and complications of viscosupplementation therapy in patients with ankle OA.
\end{abstract}

Keywords Osteoarthritis - Hyaluronic acid - Ankle joint · Viscosupplementation

S.-F. Sun $(\bowtie) \cdot$ W.-L. Chen

Department of Physical Medicine and Rehabilitation, Veterans General Hospital, Kaohsiung, No 386, Ta-Chung 1st Road,

Kaohsiung 813, Taiwan

e-mail:sfsun.tw@yahoo.com.tw

S.-F. Sun

National Yang-Ming University School of Medicine, Taipei,

Taiwan

\section{Y.-J. Chou}

Department of Orthopedic Surgery, Veterans General Hospital, Kaohsiung, Kaohsiung, Taiwan

C.-W. Hsu

Department of Internal Medicine, Veterans General Hospital, Kaohsiung, Taiwan

\section{Introduction}

Osteoarthritis (OA) is a common progressive degenerative joint disease with multiple etiologies, but similar biological, morphological, and clinical outcomes [1,2]. Individuals with OA might suffer from pain, muscle weakness, loss of joint range of motion, and increasing disability. The disease process of $\mathrm{OA}$ is characterized by the progressive erosion of articular cartilage, leading to joint space narrowing, subchondral sclerosis, subchondral cysts, synovial inflammation, and marginal osteophyte formation [3]. Traditional treatments for OA include simple analgesics, nonsteroidal anti-inflammatory drugs (NSAIDs), intra-articular corticosteroid injections, physiotherapy, activity modification, weight reduction, orthotics, and surgery [4]. Prior to surgical management of OA, which is expensive, painful, and not risk-free, all other treatment options should be fully exploited. Taking into consideration that chronic use of some oral pain medications may be contraindicated, a locally delivered therapy with no known drug interactions and an excellent safety profile is a valuable treatment option for patients.

There has been increasing interest and scientific investigation of intra-articular injection of hyaluronic acid (HA) for the treatment of OA over the past 2 decades. Creamer and Hochberg presented the treatment protocol of OA and they stated that intra-articular HA played an important role [1]. HA is a high molecular weight polysaccharide and is an important component of synovial fluid and extracellular matrix of articular cartilage. It contributes to the elasticity and viscosity of synovial fluid. HA acts as a fluid shock absorber and it helps to maintain the structural and functional characteristics of the cartilage matrix. It also inhibits the formation and release of prostaglandins, induces proteoglycan aggregation and synthesis, and modulates the inflammatory response [5, 6]. Any degradation of HA is 
associated with increased vulnerability to articular cartilage damage. OA leads to a reduction in average molecular size and concentration of $\mathrm{HA}$ in the synovial fluid [6-8]. According to Balazs and coworkers [9, 10], the injection of HA into osteoarthritic joints could restore the viscoelasticity of the synovial fluid, augment the flow of joint fluid, normalize endogenous hyaluronate synthesis, inhibit hyaluronate degradation, reduce joint pain, and improve joint function.

Viscosupplementation with intra-articular HA was approved by the Food and Drug Administration (FDA) in 1997. The American College of Rheumatology guidelines for the treatment of knee OA include the use of viscosupplementation [11]. The Orthopedic Consensus Conference made similar recommendations on the use of HA for the treatment of knee OA [12]. There are five injectable forms of HA approved by the United States FDA including Hyalgan, Supartz, Orthovisc, Synvisc, and Euflexxa. Each of these HA products differ in their origin, method of production, molecular weight, dosing instructions, biologic characteristics, and possibly clinical outcomes (Table 1). There is no consistent evidence from well-controlled clinical studies that documents the superior efficacy of one product over another.

Theoretically viscosupplementation is an approach that should apply to all synovial joints. Off-label use in degenerative arthritis of the hip, ankle, shoulder, and carpometacarpal joint of the thumb seems to be increasing and a number of recent studies have attempted to evaluate its efficacy in joints other than the knee [13-23]. The purpose of this article was to review the authors' experience, indications, clinical outcomes, and complications of viscosupplementation therapy in patients with ankle OA.

\section{Indications}

Treatment with HA is indicated for patients who are functionally limited due to osteoarthritic pain and who have failed to respond adequately to standard pharmacologic and nonpharmacologic treatment options, those who have gastrointestinal or renal intolerance to NSAIDs, and those who wish to postpone surgical intervention or are poor candidates for surgery.

Intra-articular injections of HA are contraindicated in patients with known hypersensitivity to HA preparations, avian proteins, feather and egg products, and those with active skin disease or infections in the area of the injection site. Patients with substantial venous or lymphatic stasis in the legs, bleeding disorder or treatment with anticoagulants are relatively contraindicated. HA is not recommended to pregnant women, lactating women, and children under 18 , because the safety and effectiveness have not been established.

The ideal candidate for intra-articular HA has yet to be clearly defined. We reported the results of a controlled study that patients with Kellgren-Lawrence grade I and II ankle OA had good response to viscosupplementation (grade 1, doubtful narrowing of joint space and possible osteophytic lipping; grade 2, definite osteophytes and possible narrowing of joint space) [16, 24]. This suggested that viscosupplementation was effective in mild to moderate ankle OA. Whether severe cases would likely respond to viscosupplementation remained unknown. Theoretically, patients with severe OA might have a poor and shorter response. Because the numbers of patients studied were relatively small, the results were not analyzed on the basis of disease severity, cause of OA, or preinjection functional levels. These factors might have a role in determining the candidates who would benefit most from this treatment and might help determine the best overall treatment plan. Currently, we are recruiting patients with higher X-ray severity grades in a clinical trial to see whether intraarticular HA injections can improve ankle pain and function in severely obliterated ankle joints. As the treatment group increases, hopefully we could better elucidate favorable patient response factors and identify patients who would benefit most from ankle viscosupplementation.

Table 1 Characteristics of five hyaluronans

\begin{tabular}{|c|c|c|c|c|c|}
\hline Product & Origin (method of production) & $\begin{array}{l}\text { Molecular } \\
\text { weight }(\mathrm{kd})\end{array}$ & $\begin{array}{l}\text { Amount per } \\
\text { injection }(\mathrm{ml})\end{array}$ & $\begin{array}{l}\text { Active ingredients per } \\
\text { injection }\end{array}$ & $\begin{array}{l}\text { Number of injections } \\
\text { per cycle }\end{array}$ \\
\hline Hylagan & Rooster combs (naturally derived) & $500-730$ & 2 & $20 \mathrm{mg}$ sodium hyaluronate & 3 or 5 weekly \\
\hline Supartz & Rooster combs (naturally derived) & $620-1170$ & 2.5 & $25 \mathrm{mg}$ sodium hyaluronate & 5 weekly \\
\hline Orthovisc & Rooster combs (naturally derived) & $1000-2900$ & 2 & $30 \mathrm{mg}$ sodium hyaluronate & 3 or 4 weekly \\
\hline Synvisc & $\begin{array}{l}\text { Rooster combs (chemically } \\
\text { modified } \\
\text { or cross-linked) }\end{array}$ & $\begin{array}{l}80 \%: 6000 ; 20 \%: \\
\quad>6000\end{array}$ & 2 & $\begin{array}{l}16 \mathrm{mg} \text { sodium hyaluronate } \\
\text { derivative }\end{array}$ & 3 weekly \\
\hline Euflexxa & $\begin{array}{l}\text { Bacterial fermentation (naturally } \\
\text { derived) }\end{array}$ & $2600-3400$ & 2 & $20 \mathrm{mg}$ sodium hyaluronate & 3 weekly \\
\hline
\end{tabular}




\section{Clinical outcomes}

To date there is only limited published literature on viscosupplementation therapy for ankle OA. Two recent studies seem to show efficacy on ankle pain and function (Table 2) [15, 16]. Salk et al. performed a randomized, double-blind, saline solution-controlled trial of intra-articular injection of sodium hyaluronate for the treatment of ankle OA. Both groups had significant improvement in the ankle osteoarthritis score at 6 months. However, more of the patients in the hyaluronate group had $>30$ points of improvement on the ankle osteoarthritis score as compared with the baseline value [15]. This is the first controlled study to show a benefit with HA in the treatment of ankle $\mathrm{OA}$ and is consistent with previous published studies using $\mathrm{HA}$ in the knee.

Our group performed a prospective, controlled study in patients with unilateral ankle OA and we concluded similarly that a regimen of 5 weekly intra-articular injection of sodium hyaluronate was safe and efficacious in the areas of pain and ankle function [16]. The patients' satisfaction rate was high with only relatively few local adverse events. These effects were rapid at 1 week post the fifth injection and could last for 6 months or more. One limitation in this trial includes the absence of a control group, thus the placebo effects associated with joint injections per se were not analyzed.

\section{Complications}

The safety of viscosupplementation has been well documented in clinical trials and practice. Because there are no known hyaluronate-medication interactions, it is a good option for patients on multiple medications, particularly the elderly. Mild injection-site pain and swelling are the most common adverse events in the injected joints. These reactions are usually transient, lasting 1 or 2 days and generally respond well to local modalities and NSAIDs. Other adverse events included rash, muscle cramps, dizziness, nausea, headache, local ecchymosis, and pruritus. The overall incidence of adverse reactions has been reported to be approximately $1-4 \%$ per injection [25-30]. Rare cases of calcium pyrophosphate dehydrate arthritis or pseudogout flares after both sodium hyaluronate and Synvisc have been reported [31]. There is growing evidence that Synvisc may be associated with an adverse event termed pseudosepsis or a severe acute inflammatory reaction [32-34]. Pseudosepsis presents as a severe inflammatory process of the joint, with a large effusion, and significant pain occurring within 1 to 3 days of the injection. It requires symptomatic treatment, including use of modalities, activity modification, analgesics, and NSAIDs. Once infection has been excluded, intra-articular steroids may be of value [32, 34].

No systemic adverse events were reported in the literature relating to ankle viscosupplementation. In the study by Salk et al., injection site pain was noted in 5 (29\%) of the 17 patients including 3 in HA group and 2 in the saline solution group, with no significant difference between the groups. This injection site pain typically lasted no more than 3 days.

In our ankle OA trial, we reported that local pain and erythema at the injection site occurred in 5 of 75 patients. It was mild and resolved within $48 \mathrm{~h}$ without sequela in all cases. The adverse reaction rate was $5.3 \%$ per injection and $6.7 \%$ per patient. The occurrence of adverse events was
Table 2 Clinical trials of viscosupplementation in ankle $\mathrm{OA}$

\begin{tabular}{|c|c|c|c|c|}
\hline $\begin{array}{l}\text { Author } \\
\text { date }\end{array}$ & Viscosupplement & $\begin{array}{l}\text { Study patients } \\
\text { in two different } \\
\text { locations }\end{array}$ & Outcome measures & Outcomes \\
\hline $\begin{array}{l}\text { Salk et al. } \\
\quad(2006) \\
{[15]}\end{array}$ & $\begin{array}{l}\text { Hyalgan } \\
\quad \text { (five injections) } \\
1 \mathrm{ml} \text { per injection }\end{array}$ & $\begin{array}{l}17 \\
9 \text { HA } \\
8 \text { placebo }\end{array}$ & $\begin{array}{l}\text { AOS } \\
\text { WOMAC pain domain } \\
\text { Ankle ROM } \\
\text { QOL (EQ-5D, SF-12) } \\
\text { Rescue medication table } \\
\text { count }\end{array}$ & $\begin{array}{l}\text { Safe and efficacious at } \\
6 \text { months } \\
\text { Saline solution group } \\
\text { also demonstrated a } \\
\text { significant clinical } \\
\text { benefit, albeit to a } \\
\text { lesser degree }\end{array}$ \\
\hline $\begin{array}{l}\text { Sun et al. } \\
\quad(2006) \\
{[16]}\end{array}$ & $\begin{array}{l}\text { Supartz (five } \\
\text { injections) } \\
2.5 \mathrm{ml} \text { per } \\
\text { injection }\end{array}$ & $\begin{array}{l}75 \\
75 \text { HA } \\
\text { No placebo }\end{array}$ & $\begin{array}{l}\text { AOS } \\
\text { AOFAS ankle/hindfoot score } \\
\text { Ankle ROM } \\
\text { Patients' global satisfaction } \\
\text { Rescue medication table } \\
\text { count }\end{array}$ & $\begin{array}{l}\text { Safe and efficacious, } \\
\text { effects rapid at } \\
1 \text { week post the fifth } \\
\text { injection, lasting for } \\
6 \text { months or more } \\
\text { High patients' } \\
\text { satisfaction rate. } \\
\text { Significant reduction in } \\
\text { medication } \\
\text { consumption }\end{array}$ \\
\hline
\end{tabular}


difficult to predict. They sometimes occurred after several injections without any reaction previously, and sometimes they did not occur in subsequent injections. Interestingly, we found that local adverse reactions did not predict treatment failure. All of these patient received subsequent injections and they still improved clinically and reported high satisfaction.

\section{Injection technique}

Intra-articular injection of the ankle joint is easy to perform and requires no radiologic guidance.

We position the patient in the supine position, with the knee flexed and the foot flat on the plinth. The ankle is placed in a degree of plantarflexion, which opens the anterior aspect of the joint. We identify the space between the anterior border of the medial malleolus and the medial border of the tibialis anterior tendon and palpate this space for the articulation of the talus and tibia to locate a suitable entry point into the ankle joint. An alternative point of entry may also be made at the upper, inner aspect of either malleolus. The injections are performed in aseptic conditions. We insert an 18-22 G needle into the identified space and direct posterolaterally to run parallel to the upper surface of the talus, which is slightly convex (Figs. 1 and 2). Joint effusion, if present, should be aspirated before injection to prevent dilution of the viscosupplement. Excessive weight bearing and strenuous activity are discouraged for $48 \mathrm{~h}$ after each injection.

\section{Further studies needed}

Despite the fact that HA has been proposed as a useful treatment of symptomatic knee OA, its role in the treatment of ankle OA is still not clear, due fundamentally to the

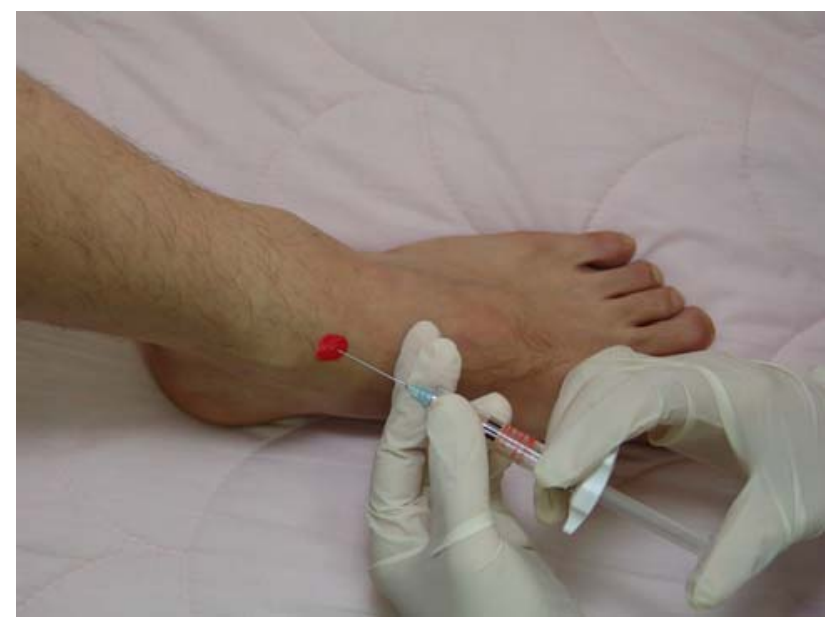

Fig. 1 Ankle joint injection

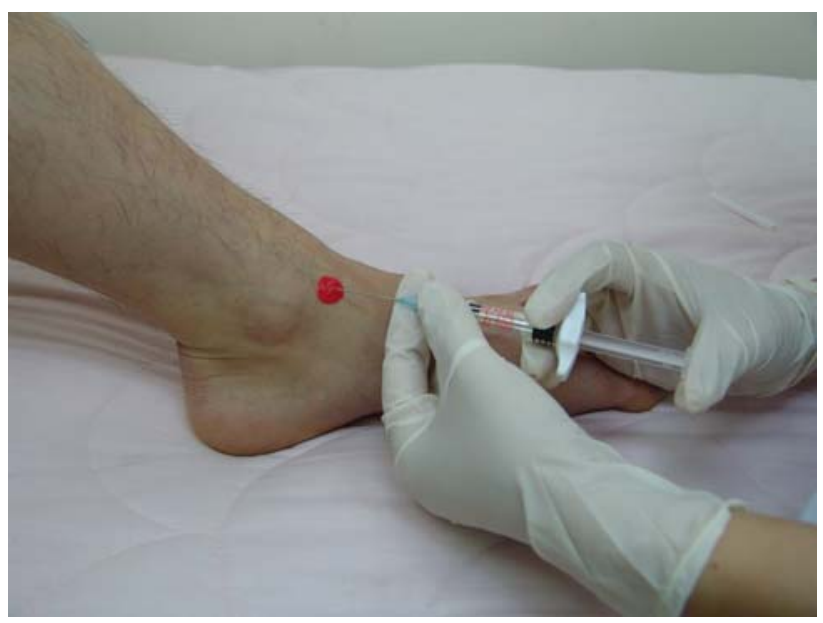

Fig. 2 Ankle joint injection

dearth of studies and to the methodological limitations of those that have been published. The exact mechanisms of viscosupplementation action on osteoarthritic joints are uncertain. Although recent attention has focused on the disease-modifying potential of HA, especially the chondroprotective mechanism, definitive evidence is still lacking. We believe that HA can be used as an adjunct therapy, after failure of one or more courses of oral pain medications, or perhaps as a first choice in the treatment of ankle OA before prescription of pain medications. It is important to understand the distinct mechanisms of HA to help appropriately place viscosupplementation into the physicians' treatment algorithms.

Future studies regarding optimal dosing regimen; optimal number of injections in a course of treatment; favorable prognostic factors; effectiveness and safety of repeated courses of therapy; different concentration and molecular weight options for ankle viscosupplementation; as well as the biochemical, morphologic, and histopathologic effects on cartilage are warranted. Cost-effectiveness needs to be addressed. The robust placebo effect deserves special attention when critically interpreting outcome data. Comparison studies or combination therapies with other treatment options, such as intra-articular steroid injections, NSAIDs, and therapeutic exercise, are also needed.

We hoped that this review will stimulate interest in research to assess the potential role of viscosupplementation in treating ankle OA and more research is needed to demonstrate its true benefit before this treatment option will be fully accepted.

\section{Conclusion}

The published data suggest that viscosupplementation may be a safe and effective method in the treatment of ankle 
OA. However, the limited number of patients with ankle OA enrolled in clinical trials precludes any definitive conclusion about its safety and efficacy. To date, ankle viscosupplementation should only be used under careful supervision by the clinician. Many uncertainties on the use of HA remain. More studies with larger patient populations are required before viscosupplementation should be included into the treatment paradigm for patients with ankle OA.

Open Access This article is distributed under the terms of the Creative Commons Attribution Noncommercial License which permits any noncommercial use, distribution, and reproduction in any medium, provided the original author(s) and source are credited.

\section{References}

1. Creamer P, Hochberg MC. Osteoarthritis. Lancet. 1997;350:5038.

2. Felson DT, Lawrence RC, Dieppe PA, et al. Osteoarthritis: new insights. Part 1: the disease and its risk factors. Ann Intern Med. 2000;133:635-46.

3. Buckwalter JA, Mankin HJ. Articular cartilage: degeneration and osteoarthritis, repair, regeneration, and transplantation. Instr Course Lect. 1998;47:487-504.

4. Adams ME. An analysis of clinical studies of the use of cross linked hyaluronan, hylan, in the treatment of osteoarthritis. J Rheumatol Suppl. 1993;39:16-8.

5. Frizziero L, Govoni E, Bachin P. Intraarticular hyaluronic acid in the treatment of osteoarthritis of the knee: clinical and morphological study. Clin Exp Rheumatol. 1998;16:441-9.

6. Adams ME, Atkinson MH, Lussier AJ, et al. The role of viscosupplementation with hylan G-F 20 (Synvisc) in the treatment of osteoarthritis of the knee: a Canadian multicenter trial comparing hylan G-F 20 alone, hylan G-F 20 with non-steroidal antiinflammatory drugs (NSAIDs) and NSAIDs alone. Osteoarthritis Cartilage. 1995;3(4):213-25.

7. Belcher C, Yaqub R, Fawthrop F, Bayliss M, et al. Synovial fluid chondroitin and keratan sulphate epitopes, glycosaminoglycans, and hyaluronan in arthritic and normal knees. Ann Rheum Dis. 1997;56:299-307.

8. Engström-Laurent A. Hyaluronan in joint disease. J Int Med. 1997;242:57-60.

9. Balazs EA, Denlinger JL. Viscosupplementation: a new concept in the treatment of osteoarthritis. J Rheumatol Suppl. 1993;39: 4-9.

10. Rydell N, Balazs EA. Effect of intra-articular injection of hyaluronic acid on the clinical symptoms of osteoarthritis and on granulation tissue formation. Clin Orthop. 1971;80:25-32.

11. American College of Rheumatology Subcommittee on Osteoarthritis Guidelines. Recommendations for the medical management of osteoarthritis of the hip and knee 2000 update. Arthritis Rheum. 2000;43:1905-15.

12. Kelly MA, Goldberg VM, Healy WL, et al. Osteoarthritis and beyond: a consensus on the past, present, and future of hyaluronans in orthopedics. Orthopedics. 2003;26:1064-81.

13. Pleimann JH, Davis WH, Cohen BE, et al. Viscosupplementation for the arthritic ankle. Foot Ankle Clin. 2002;7(3):489-94.

14. Wang CW, Gao LH, Jin XY, et al. Clinical study of sodium hyaluronate in supplementary treatment of comminuted fracture of ankle. Zhongguo Xiu Fu Chong Jian Wai Ke Za Zhi. 2002; 16(1):21-2.
15. Salk RS, Chang TJ, D'Costa WF, et al. Sodium hyaluronate in the treatment of osteoarthritis of the ankle: a controlled, randomized, double-blind pilot study. J Bone Jt Surg Am. 2006;88:295-302.

16. Sun SF, Chou YJ, Hsu CW, et al. Efficacy of intra-articular hyaluronic acid in patients with osteoarthritis of the ankle: a prospective study. Osteoarthritis Cartilage. 2006;14(9):867-74.

17. Conrozier T, Bertin P, Mathieu P, et al. Intra-articular injections of hylan G-F 20 in patients with symptomatic hip osteoarthritis: an open label, multicentre, pilot study. Clin Exp Rheumatol. 2003;21:605-10

18. Migliore A, Tormenta S, Martin LSM, et al. Open pilot study of ultrasound-guided intra-articular injection of hylan G-F 20 (Synvisc) in the treatment of symptomatic hip osteoarthritis. Clin Rheumatol. 2005;24:285-9.

19. Tikiz C, Unlu Z, Sener A, et al. Comparison of efficacy of lower and higher molecular weight viscosupplementation in the treatment of hip osteoarthritis. Clin Rheumatol. 2005;24:244-50.

20. Labbe M, Ridgeland E, Savoie FH, et al. The short-term efficacy of hyaluronic acid injections for the treatment of degenerative arthrosis of the shoulder. Arthroscopy. 2003;19:S13-8.

21. Marshall KW. Intra-articular hyaluronan therapy. Foot Ankle Clin N Am. 2003;8:221-32.

22. Clarke S, Lock V, Duddy J, et al. Intra-articular hylan G-F 20 in the management of patellofemoral osteoarthritis of the knee. The Knee. 2005;12:57-62.

23. Roux C, Fontas E, Breuil V, et al. Injection of intra-articular sodium hyaluronidate (Sinovial) into the carpometacarpal joint of the thumb $(\mathrm{CMC} 1)$ in osteoarthritis. A prospective evaluation of efficacy. Jt Bone Spine. 2007;74(4):368-72.

24. Kellgren JH, Lawrence JS. Atlas of standard radiographs: the epidemiology of chronic rheumatism, vol. 2. Oxford, UK: Blackwell Scientific Publications; 1963.

25. Hammesfahr JF, Knopf AB, Stitik T. Safety of intra-articular hyaluronates for pain associated with osteoarthritis of the knee. Am J Orthop. 2003;32:277-83.

26. Watterson JR, Esdaile JM. Viscosupplementation: therapeutic mechanisms and clinical potential in osteoarthritis of the knee. $\mathrm{J}$ Am Acad Orthop Surg. 2000;8:277-84.

27. Lussier A, Cividino AA, McFarlane CA, et al. Viscosupplementation with hylan for the treatment of osteoarthritis: findings from a clinical practice in Canada. J Rheumatol. 1996;23:157985.

28. Kemper F, Gebhardt U, Meng T, et al. Tolerability and short-term effectiveness of hylan G-F 20 in 4253 patients with osteoarthritis of the knee in clinical practice. Curr Med Res Opin. 2005;21:1261-9.

29. Waddell DD. The tolerability of viscosupplementation: low incidence and clinical management of local adverse events. Curr Med Res Opin. 2003;19:575-80.

30. Waddell DD, Bricker DC. Clinical experience with the effectiveness and tolerability of hylan G-F 20 in 1047 patients with osteoarthritis of the knee. J Knee Surg. 2006;19:19-27.

31. Hamburger MI, Lakhanpal S, Mooar PA, et al. Intraarticular hyaluronans: a review of product-specific safety profiles. Semin Arthritis Rheum. 2003;32:296-309.

32. Goldberg VM, Coutts RD. Pseudoseptic reactions to hylan viscosupplementation: diagnosis and treatment. Clin Orthop. 2004;419:130-7.

33. Marino AA, Waddell DD, Kolomytkin OV, et al. Assessment of immunologic mechanisms for flare reactions to Synvisc. Clin Orthop. 2006;442:187-94.

34. Leopold SS, Warme WJ, Pettis PD, et al. Increased frequency of acute local reaction to intra-articular hylan G-F 20 in patients receiving more than one course of treatment. J Bone Jt Surg. 2002;84A:1619-23. 